\title{
Overexpression of MYC binding protein promotes invasion and migration in gastric cancer
}

\author{
LIJIE GONG ${ }^{1,2^{*}}$, YINGJIE XIA ${ }^{3 *}$, ZHENYUAN QIAN $^{1,4}$, JI SHI $^{5}$, JUNGANG LUO ${ }^{1}$, \\ GUANGYUAN SONG ${ }^{6 *}$, JI XU ${ }^{1,4}$ and ZAIYUAN YE ${ }^{1}$
}

${ }^{1}$ Department of Gastrointestinal and Pancreatic Surgery, Zhejiang Provincial People's Hospital;

${ }^{2}$ The Second Clinical Medical College, Zhejiang Chinese Medical University;

${ }^{3}$ Key Laboratory of Gastroenterology of Zhejiang Province, Zhejiang Provincial People's Hospital,

People's Hospital of Hangzhou Medical College; ${ }^{4}$ Department of Gastrointestinal and Pancreatic Surgery, People's Hospital of Hangzhou Medical College; ${ }^{5}$ Department of Breast and Thyroid Surgery, Tongde Hospital of Zhejiang Province; ${ }^{6}$ Zhejiang University School of Medicine, Zhejiang University, Hangzhou, Zhejiang 310000, P.R. China

Received May 22, 2017; Accepted December 8, 2017

DOI: $10.3892 / 01.2018 .7944$

\begin{abstract}
Gastric cancer (GC) is the second leading cause of cancer-associated mortality worldwide. Although the mortality rate of patients with GC has improved, it remains a significant health issue. The MYC proto-oncogene protein serves key roles in cellular proliferation, differentiation, transformation and apoptosis. Previous studies have identified the abnormal expression of MYC-binding protein (MYCBP) during tumorigenesis in multiple types of cancer. Furthermore, evidence demonstrates that the abnormal expression of MYCBP contributes to the invasion and migration of human cancer types, including colon cancer and glioma; however, its influence on GC remains unclear. In the present study, the expression of MYCBP in GC cells and tissues was analyzed by reverse transcription-quantitative polymerase chain reaction. Additionally, GC cell lines were transfected with small interfering RNAs against MYCBP or lymphoid enhancer-binding factor 1 (LEF-1) and assessed by in vitro transwell migration and invasion assays. The results indicated that the expression of MYCBP in GC cells and tissues was markedly increased compared with a normal gastric epithelial cell line and adjacent normal gastric mucosal tissues, respectively. Furthermore, MYCBP downregulation notably inhibited the
\end{abstract}

Correspondence to: Dr Ji Xu or Dr Zaiyuan Ye, Department of Gastrointestinal and Pancreatic Surgery, Zhejiang Provincial People's Hospital, People's Hospital of Hangzhou Medical College, 158 Shangtang Road, Hangzhou, Zhejiang 310000, P.R. China

E-mail: xuji120@163.com

E-mail: zaiyuanye@163.com

*Contributed equally

Key words: MYC binding protein, gastric cancer, lymphoid enhancer-binding factor 1, migration, invasion metastatic capacity of GC cells, and LEF-1 knockdown was found to downregulate the expression of MYCBP. On the basis of the findings of the present study, MYCBP may be a direct target of the $\beta$-catenin/LEF-1 pathway via binding LEF-1, and could potentially be used as a biomarker for the diagnosis and prognosis of GC.

\section{Introduction}

Gastric cancer (GC) is the most common gastrointestinal malignant tumor, although its incidence rates vary across different countries and regions. A high incidence of GC is observed in individuals between 40 and 60 years of age (1). Each year, 900,000 new cases of GC are diagnosed, while $\sim 700,000$ patients succumb to GC globally (2), with half of these mortalities occurring in Asia. The incidence rate in males is approximately two-fold higher than that in females (3). The pathogenesis of GC is unclear and lacks specific clinical manifestations, and therefore the rate of early diagnosis is low (4). Regional variations in GC incidence reflect differences in dietary patterns, food storage, and the availability of fresh produce, as well as the prevalence of Helicobacter pylori infection (5). Surgical resection remains the first-choice treatment modality for GC (6). However, even with a combination of surgical and chemotherapeutic treatments, the 5-year overall survival rate for patients with advanced-stage GC is $\leq 20 \%$ (2).

The MYC proto-oncogene protein (hereafter MYC) serves key roles in the proliferation (7), cell cycle (8), differentiation and apoptosis of cells $(9,10)$. However, abnormal expression of MYC has been implicated in almost all human tumors (11). Activation of the MYC gene is frequently associated with the progression of tumors, poor patient prognosis and malignant properties, including increased mobility, and invasive and metastatic capacities (12). The expression and functional regulation of the MYC gene involves a variety of mechanisms, one of which is based on the MYC-binding protein (MYCBP) signaling pathway (13). The MYCBP gene encodes a protein of $\sim 11 \mathrm{kDa}$ which, through its $\mathrm{C}$-terminal structure, can bind the 
MYC N-terminal region, thereby activating MYC to promote tumorigenesis (14). MYCBP has been identified as a target of $\beta$-catenin/T cell factor (TCF)/lymphoid enhancer-binding factor (LEF) transcriptional regulation in colon carcinoma (15).

Recently, researchers have demonstrated that $30-50 \%$ of GC cases are associated with the abnormal activation of the Wnt/ $\beta$-catenin signaling pathways $(16,17)$. The TCF/LEF axis serves a crucial role in the Wnt/ $\beta$-catenin signaling pathway (18). A previous study observed that the transcription of a series of target genes within the Wnt/ $\beta$-catenin signaling pathway was activated following the formation of a $\mathrm{TCF} / \mathrm{LEF} / \beta$-catenin complex in the nucleus, which regulated cellular biological activities and promoted the migratory and invasive abilities of tumor cells (19). Recent studies have also indicated that MYCBP is associated with tumorigenesis in a variety of tumor types, including colon cancer and glioma $(20,21)$. However, the potential role of MYCBP in promoting the growth of GC has, to the best of our knowledge, not been reported. In the present study, the role of MYCBP as a potential biomarker for the diagnosis and prognosis of GC was evaluated.

\section{Materials and methods}

Cell culture. The human GC SGC-7901, MKN-45, AGS and BGC-823 cell lines, and the human gastric mucosal epithelial GES-1 cell line were provided by the Cell Bank of the Shanghai Institute of Cell Biology (Shanghai, China). All cells were cultured in RPMI 1640 medium (Hyclone; GE Healthcare Life Sciences, Logan, UT, USA) containing $10 \%$ fetal bovine serum (FBS; Hyclone; GE Healthcare Life Sciences, Logan, UT, USA) and antibiotics (100 U/ml streptomycin and $100 \mathrm{U} / \mathrm{ml}$ penicillin; Hyclone; GE Healthcare Life Sciences, Logan, UT, USA), and maintained at $37^{\circ} \mathrm{C}$ under $5 \% \mathrm{CO}_{2}$ and $100 \%$ humidity atmosphere. Cells were passaged at $80 \%$ confluency using $0.02 \%$ EDTA/0.25\% trypsin for 3-5 min.

Clinical samples. A total of 77 fresh specimens from patients via GC resection (aged 42-78 years, median age of 64 years, 45 male and 32 female patients) were acquired from Zhejiang Provincial People's Hospital (Hangzhou, China) between January and December 2015 , and stored at $-80^{\circ} \mathrm{C}$. Paired adjacent non-cancerous tissues were also obtained from patients. None of the patients had received radiotherapy or chemotherapy prior to surgery. The diagnosis of all gastric cancer patients depended on the results of pathological sections. All samples were verified by three pathologists at the Department of Pathology, Zhejiang Provincial People's Hospital. Tumor grade was determined according to various classifications of tumors [Tumor-Node-Metastasis (22)]. Written informed consent was obtained from all patients prior to participation and the study was approved by the Ethics Committee of Zhejiang Provincial People's Hospital. All procedures performed involving human participants were conducted in accordance with the ethical standards of the institutional and/or national research committee, and with The 1964 Helsinki Declaration and its later amendments.

Reverse transcription-quantitative polymerase chain reaction (RT-qPCR). Total RNA was extracted from the cell lines and fresh specimens using TRIzol reagent (Thermo Fisher Scientific, Inc., Waltham, MA, USA), according to the manufacturer's protocol. cDNA was prepared using a Superscript III cDNA Synthesis kit (Takara Bio, Inc., Otsu, Japan) following the manufacturer's protocol. qPCR was performed using FastStart Essential DNA Green Master mix (Roche Diagnostics, Basel, Switzerland) with miRNA-specific primers. GAPDH was used as the endogenous control. The specific primers used were as follows: GAPDH forward, 5'-TGAAGGTCGGAG TCAACGG-3' and reverse, 5'-CTGGAAGATGGTGATGGG ATT-3'; MYCBP were forward, 5'-TGGCACCTGTTGGAG ACTATG-3' and reverse, 5'-CACCAGCCATAGCCACA TTC-3'. PCR thermocycling conditions were as follows: $95^{\circ} \mathrm{C}$ for $10 \mathrm{~min}$, followed by 40 cycles of $95^{\circ} \mathrm{C}$ for $10 \mathrm{sec}, 58^{\circ} \mathrm{C}$ for $10 \mathrm{sec}$ and $72^{\circ} \mathrm{C}$ for $10 \mathrm{sec}$. At the end of the PCR cycles, melting curve analysis was performed. MYCBP expression levels in tumor tissues and cell lines were compared with those in the matched normal tissues and normal gastric cells, respectively, and relative expression levels were calculated using the $2^{-\Delta \Delta \mathrm{Cq}}$ method (23).

MYCBP small interfering RNA (siRNA) and LEF-1 siRNA transfection. MKN-45 cells exhibited a relatively high level of MYCBP expression compared with the normal gastric cell line GES-1 and the other GC cell lines, and thus were used in the transfection assays. The MKN-45 cells were cultured in 6-well plates (plated at $5.0 \times 10^{5}$ cells/well) for $24 \mathrm{~h}$ prior to transfection. Subsequently, MYCBP siRNA (20 $\mu \mathrm{mol} / \mathrm{l}$; Guangzhou RiboBio Co., Ltd., Guangzhou, China) and LEF-1 siRNA (20 $\mu \mathrm{mol} / \mathrm{l}$; Guangzhou RiboBio Co., Ltd.) were individually transfected into the MKN-45 cells. Negative control siRNA (Guangzhou RiboBio Co., Ltd.) was used to establish a negative control group in parallel. The specific sequences used were as follows: MYCBP siRNA forward, 5'-AAUCCA AAGCACUGUUAGGUU-3' and reverse, 5'-CCUAACAGU GCUUUGGAUUUU-3'; LEF-1 siRNA forward, 5'-ACU UGAUGUCAGCUAAAUCGC-3' and reverse, 5'-GAUUUA GCUGACAUCAAGUCU-3'; NC siRNA forward, 5'-UUC UCCGAACGUGUCACGUTT-3' and reverse, 5'-ACGUGA CACGUUCGGAGAATT-3'. The two negative controls have the same siRNA sequence. Transfections were performed with Lipofectamine ${ }^{\circledR} 3000$ (Thermo Fisher Scientific, Inc.) according to the manufacturer's protocol.

Transwell assay. At $24 \mathrm{~h}$ after transfection, GC cells were used in migration and invasion assays. Transwell migration assays were performed using a Costar Transwell assay kit (Corning Incorporated, Corning, NY, USA), whereas invasion assays were performed using invasion chambers (Corning Incorporated) pre-coated with Matrigel. Cells $\left(1.5 \times 10^{5}\right.$ per well for invasion assays and $8.0 \times 10^{4}$ per well for migration assays) were seeded in the upper chamber with FBS-free RPMI-1640 medium, and RPMI-1640 medium containing 30\% FBS was added to the lower chamber. After 24 or $48 \mathrm{~h}$ of incubation at $37^{\circ} \mathrm{C}$ in an atmosphere of $5 \% \mathrm{CO}_{2}$, non-migrated or non-invaded cells were removed from the upper surfaces of the transwell membranes with a cotton swab, and the migrated or invaded cells on the lower membrane surfaces were fixed by 95\% ethyl alcohol for $10 \mathrm{~min}$ at room temperature and stained with hematoxylin and eosin for $15 \mathrm{~min}$ at room temperature 


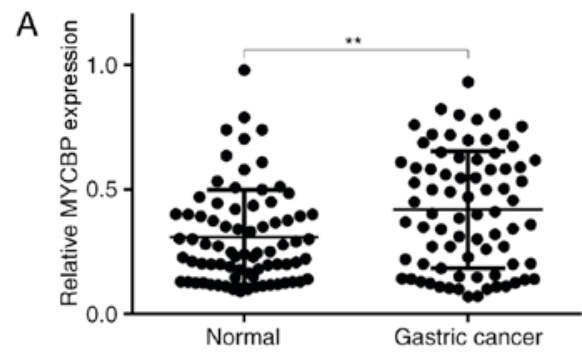

C

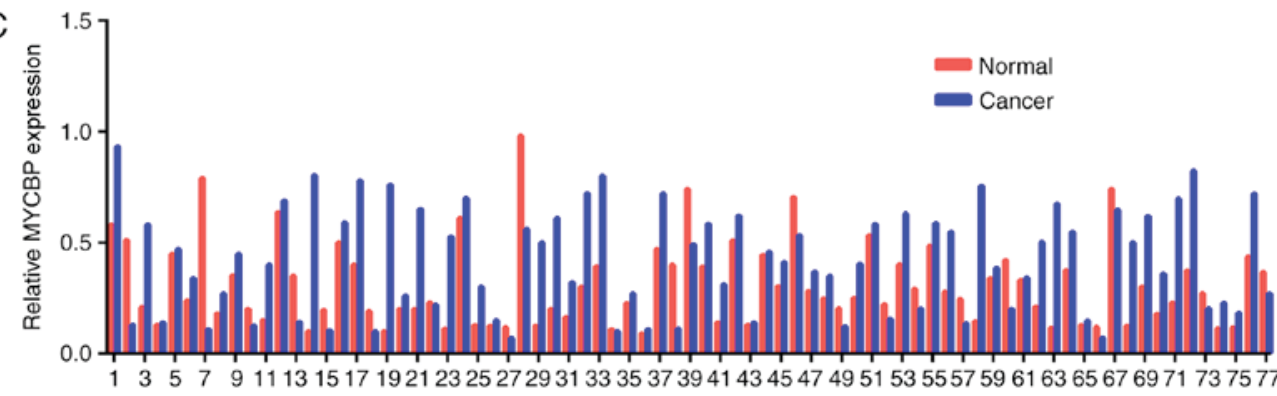

B

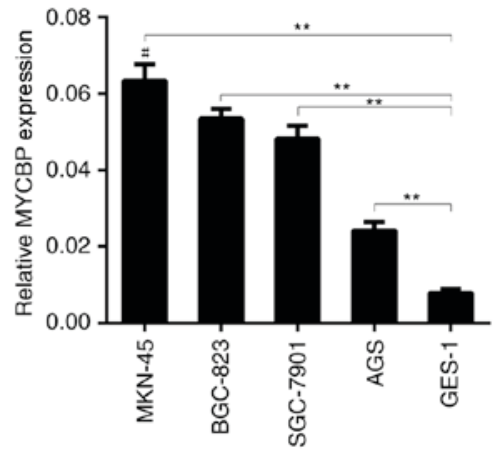

Figure 1. Expression of MYCBP in GC tissues and cells. (A) For the majority of cases, the mean expression level of MYCBP in the GC tissue samples was higher than that in the pair-matched adjacent normal tissues, as measured by RT-qPCR. (B) MYCBP expression levels were also detected in GC cell lines by RT-qPCR. The relative levels of MYCBP expression in the GC cell lines were markedly higher than that in normal gastric GES-1 cells. (C) Relative MYCBP expression in GC and adjacent normal tissues from each patient. ${ }^{*} \mathrm{P}<0.05$ (vs. BGC-823 or SGC-7901 or AGS), ${ }^{* *} \mathrm{P}<0.01$. GC, gastric cancer; MYCBP, MYC-binding protein; RT-qPCR, reverse transcription-polymerase chain reaction.

prior to imaging and counting under high-power magnification (x200; Olympus IX71 live cell imaging fluorescence microscope; Olympus, Tokyo, Japan).

Cell cycle and apoptosis assay. At $24 \mathrm{~h}$ following transfection with MYCBP siRNA, LEF-1 siRNA or control siRNA, MKN45 cells were washed with PBS and fixed with $70 \%$ ethanol for $>12 \mathrm{~h}$ at $4{ }^{\circ} \mathrm{C}$. Following centrifugation $(100 \mathrm{x} \mathrm{g})$ at room temperature for $3 \mathrm{~min}$, cells were incubated with $500 \mu \mathrm{l}$ propidium iodide (PI; Beyotime Institute of Biotechnology, Haimen, China) staining solution for $30 \mathrm{~min}$ in the dark. The cell cycle distribution was analyzed by flow cytometry (FACSCalibur flow cytometer with CellQuest software (version. 5.1; BD Biosciences, Franklin Lakes, NJ, USA). Additionally, an Annexin V/PI Apoptosis Detection kit (Beyotime Institute of Biotechnology) was used to assess cell apoptosis. Briefly, Annexin V and PI were used to label early and late apoptotic cells, respectively and, following staining for $15 \mathrm{~min}$ at room temperature, the cells were analyzed with the FACSCalibur flow cytometer and CellQuest software to detect apoptotic cells.

Western blot analysis. According to the results of the prediction of the genes interaction on the Genecards, MYCBP may be a downstream gene of LEF-1 (http://www.genecards. org/). This prediction was investigated by western blotting. In brief, MKN-45 GC cells transfected with LEF-1, MYCBP or control siRNA (Guangzhou RiboBio, Co., Ltd., Guangzhou, China) were harvested, washed and lysed with Radioimmunoprecipitation Assay buffer (Beyotime Institute of Biotechnology). Total protein concentration was measured using a bicinchoninic acid protein assay kit (Thermo Fisher Scientific, Inc.). Equivalent quantities (30-50 $\mu \mathrm{g}$ per lane) of protein were separated by $12 \%$ sodium dodecyl sulfate-polyacrylamide gel electrophoresis and transferred to polyvinylidene fluoride microporous membranes (EMD Millipore, Billerica, MA, USA). Membranes were blocked with $5 \%$ non-fat milk in Tris-buffered saline for $2 \mathrm{~h}$ at room temperature, and then incubated overnight at $4{ }^{\circ} \mathrm{C}$ with primary antibodies against LEF-1 (1:1,000; cat. no. 14972-1-AP; ProteinTech Group, Inc., Chicago, IL, USA), MYCBP (1:1,000; cat. no. 12022-1-AP; ProteinTech Group, Inc.), MYC (1:1,000; cat. no. 10828-1-AP; ProteinTech Group, Inc.) and GAPDH (1:1,000; cat. no. 10828-1-AP; ProteinTech Group, Inc.) at the dilutions specified by the manufacturer. The membranes were washed three times with TBS with Tween-20 (1:1,000) and incubated with corresponding horseradish peroxidase (HRP)-conjugated secondary antibodies (cat. no. HA1001; HuaBio, Hangzhou, China) at 1:1,000 dilution for $1 \mathrm{~h}$. Bound secondary antibodies were detected using an enhanced chemiluminescence system (Wuhan Sanying Biotechnology, Wuhan, China).

Statistical analysis. Statistical analysis was performed using SPSS version 22.0 (IBM Corp., Armonk, NY, USA). P<0.05 was considered to indicate a statistically significant difference. The MYCBP levels determined by qPCR were expressed as the mean \pm standard deviation. The means of normally distributed results were compared by either paired Student t-tests or one-way analysis of variance, as appropriate.

\section{Results}

MYCBP expression in GC tissues. The expression of MYCBP in $77 \mathrm{GC}$ and matched adjacent normal gastric mucosal tissue samples was detected by RT-qPCR. The expression of MYCBP 

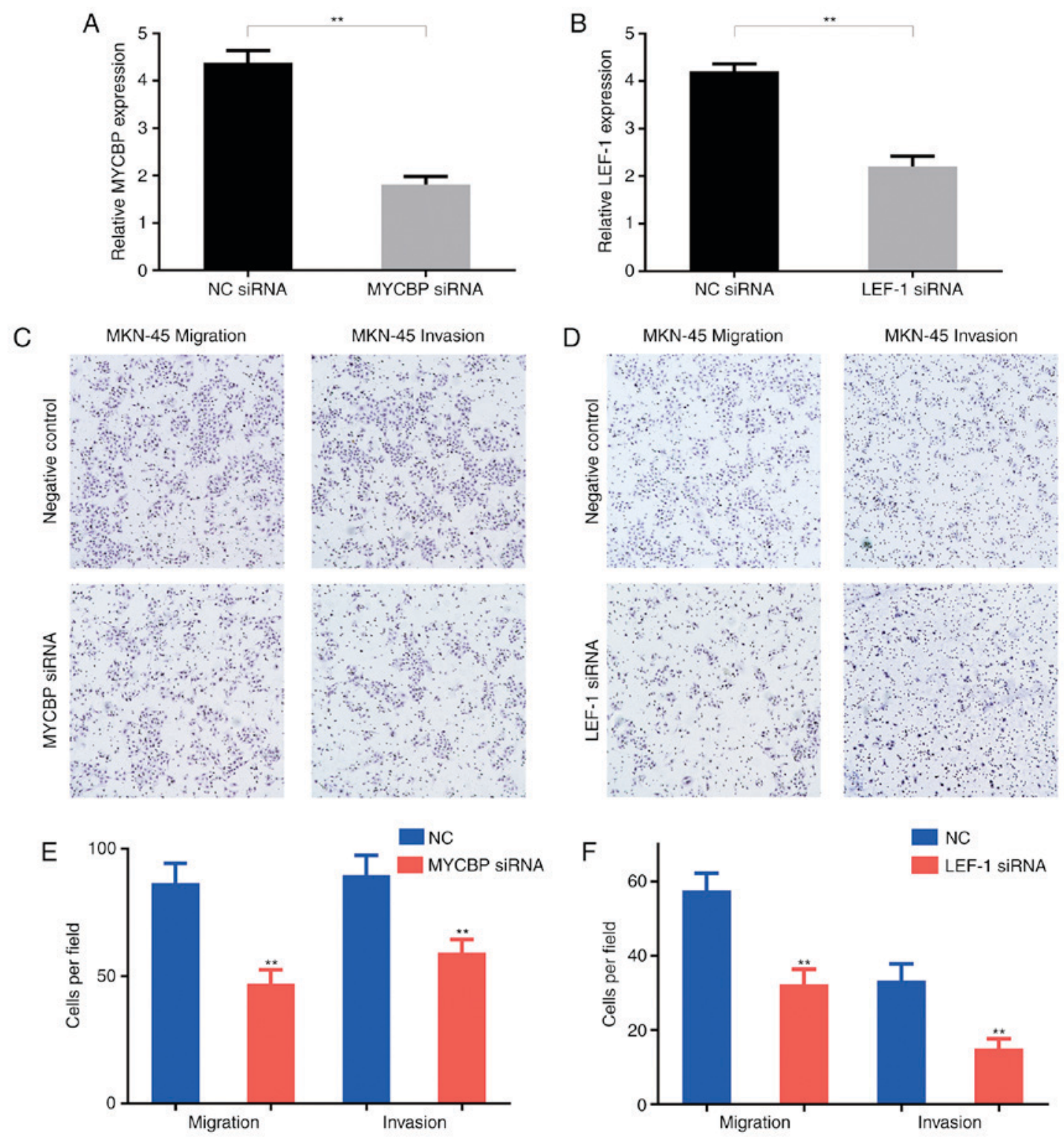

Figure 2. (A) Relative MYCBP expression levels in control and MYCBP siRNA-treated MNK45 cells. (B) Relative LEF-1 expression of siRNA in control and LEF-1 siRNA treated MNK45 cells is demonstrated. (C and D) Migration and invasion assays were performed (magnification, x200) and (E and F) quantified (C and E) GC cells (MKN-45) transfected with MYCBP siRNA exhibited decreased migration and invasion activity compared with the negative control group. (D and F) GC cells (MKN-45) transfected with LEF-1 siRNA exhibited decreased migration and invasion activity compared with the negative control group. ${ }^{* *} \mathrm{P}<0.01$ vs. NC. GC, gastric cancer; MYCBP, MYC-binding protein; LEF-1, lymphoid enhancer-binding factor 1; NC, normal control; siRNA, small interfering RNA.

in the GC tissues was significantly higher than that in the adjacent normal gastric mucosal tissues $(0.4171 \pm 0.027$ vs. $0.3076 \pm 0.022$, $\mathrm{P}<0.01$; Fig. 1A and $1 \mathrm{C}$ ), and the relative MYCBP overexpression rate was $71.4 \%$. Furthermore, in GC cells in vitro, the expression levels of MYCBP were significantly higher in the four GC cell lines (SGC-7901, MKN-45, BGC-823 and AGS) than in the GES-1 normal gastric mucosal cell line (Fig. 1B). As shown in Fig. 1C, the expression of MYCBP in each paired samples is different. Among the GC cell lines, MKN-45 exhibited a relatively higher level of MYCBP expression.

MYCBP and LEF-1 affect the invasion and migration capacities of $G C$ cells. To investigate the role of MYCBP in the development and progression of GC, MYCBP or LEF-1 siRNA were transfected into the GC cell line MKN-45, and the effects on cell migration and invasion capacities were investigated using transwell assays (Fig. 2). The expression of MYCBP and LEF-1incells transfected with MYCBP siRNA and LEF-1 siRNA was observed to be significantly inhibited $(\mathrm{P}<0.01$; Fig. 2A and B). It was observed that the numbers of MKN-45 cells invading and migrating across the transwell membrane were significantly reduced by transfection with the MYCBP siRNA or LEF-1 siRNA ( $\mathrm{P}<0.01$; Fig. $2 \mathrm{C}-\mathrm{F})$ when compared with those in the negative control group. These data indicated that MYCBP and LEF-1 affected the invasion and migration capacities of the GC cells.

Effects of MYCBP and LEF-1 on GC cell cycle and apoptosis. To investigate the role of MYCBP in GC further, the cell cycle distribution and apoptosis of MKN-45 cells was assessed following transfection with the MYCBP and LEF-1 siRNAs. According to the cell cycle assay, MKN-45 cells transfected with MYCBP 
A

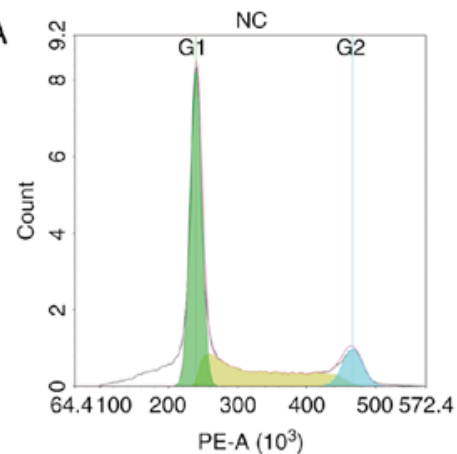

B

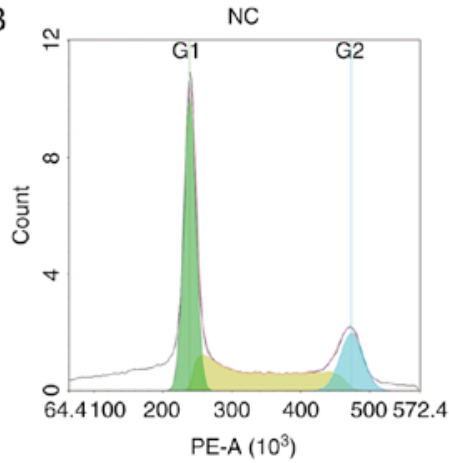

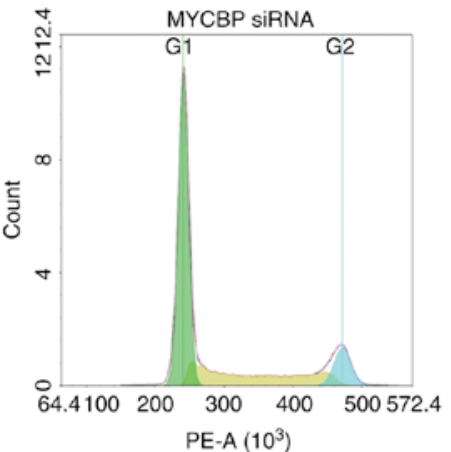
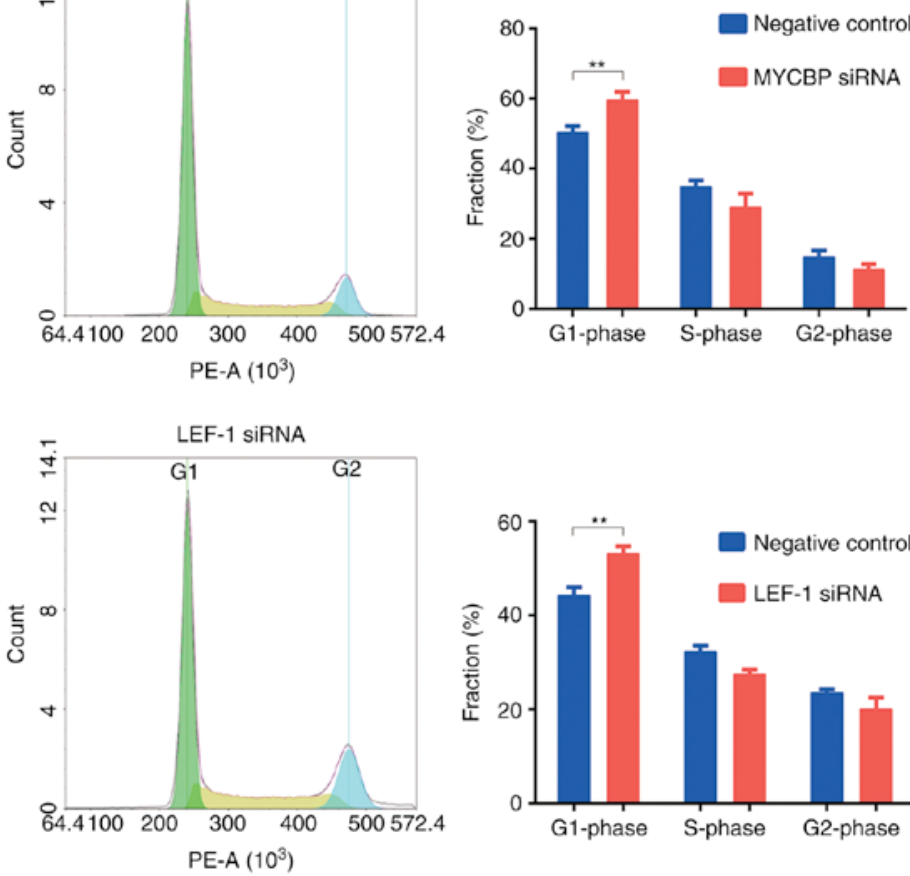

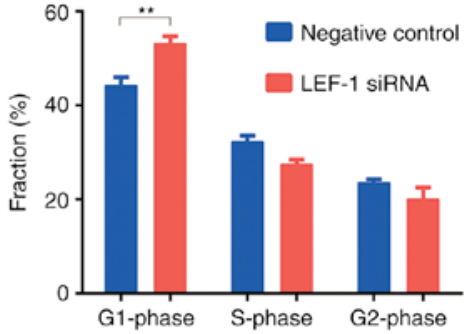

Figure 3. (A) MKN-45 cells transfected with MYCBP siRNA exhibited cell cycle arrest in the $\mathrm{G}_{1}$ phase when compared with the control group, as measured by flow cytometry. (B) MKN-45 cells transfected with LEF-1 siRNA exhibited marked arrest in the $\mathrm{G}_{1}$ phase when compared with the control group, as measured by flow cytometry. ${ }^{* *} \mathrm{P}<0.01$. GC, gastric cancer; MYCBP, MYC-binding protein; LEF-1, lymphoid enhancer-binding factor 1; NC, normal control; siRNA, small interfering RNA.

A

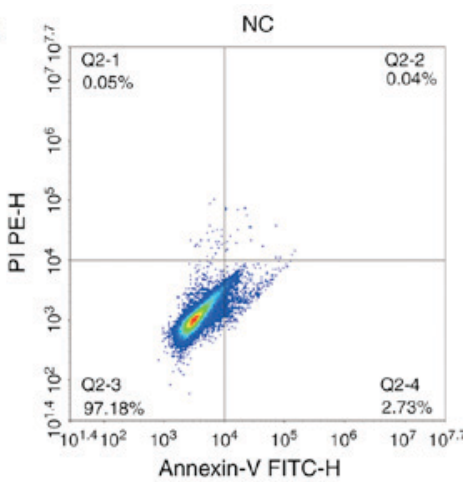

B

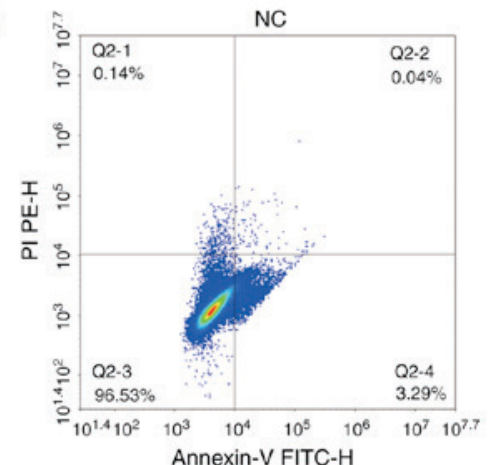

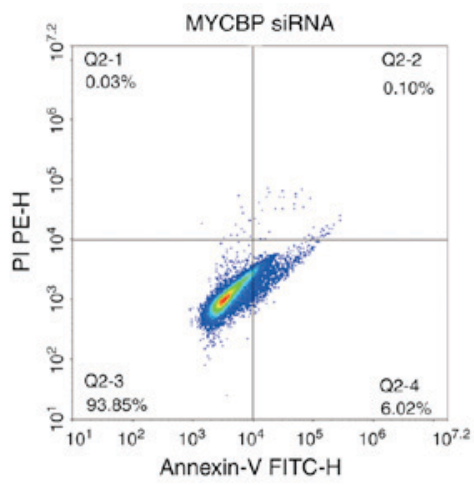
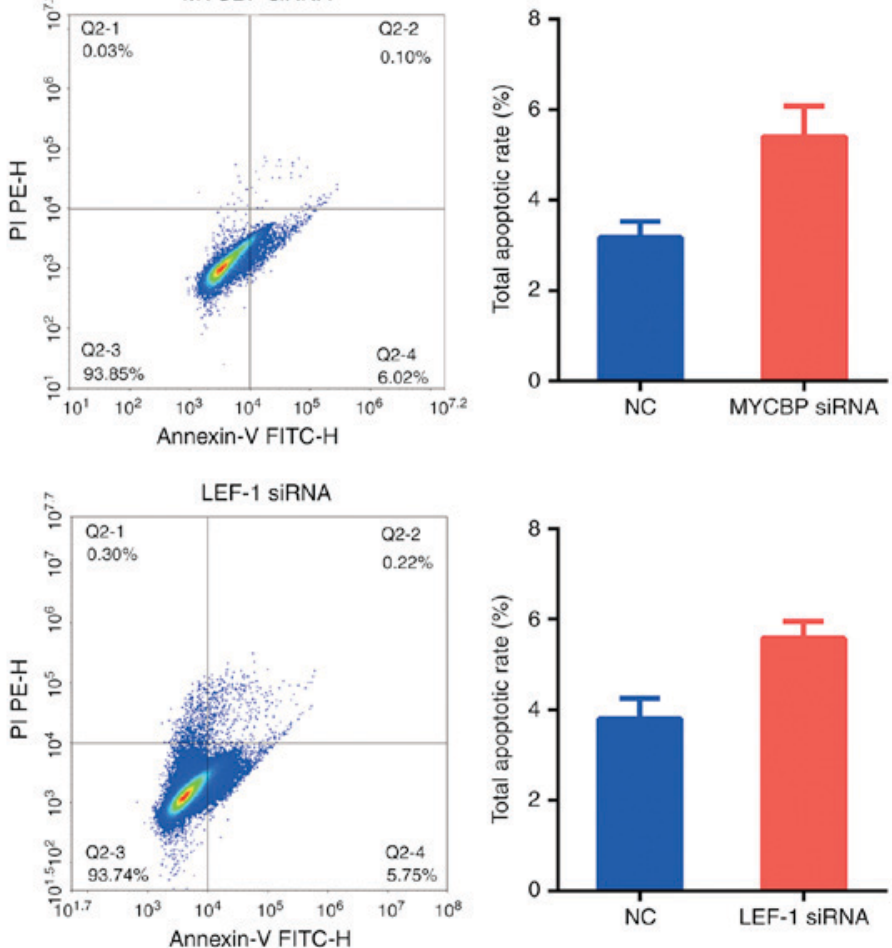

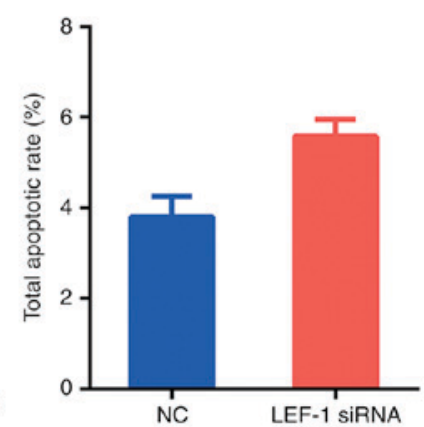

Figure 4. MKN-45 cells transfected with (A) MYCBP siRNA and (B) LEF-1 siRNA exhibited no significant difference in apoptotic rate compared with the control group. GC, gastric cancer; MYCBP, MYC-binding protein; LEF-1, lymphoid enhancer-binding factor 1; NC, normal control; siRNA, small interfering RNA.

or LEF-1 siRNA were markedly arrested in the $\mathrm{G}_{1}$ phase, with percentages of $\mathrm{G}_{1}$-phase cells of $59.6 \pm 2.3$ and $53.2 \pm 1.6 \%$, respectively, when compared with the control group $(50.4 \pm 1.8$ and $44.2 \pm 1.8 \%$, respectively; Fig. 3). However, the difference in the rate of cell apoptosis between groups was not statistically significant (Fig. 4). 


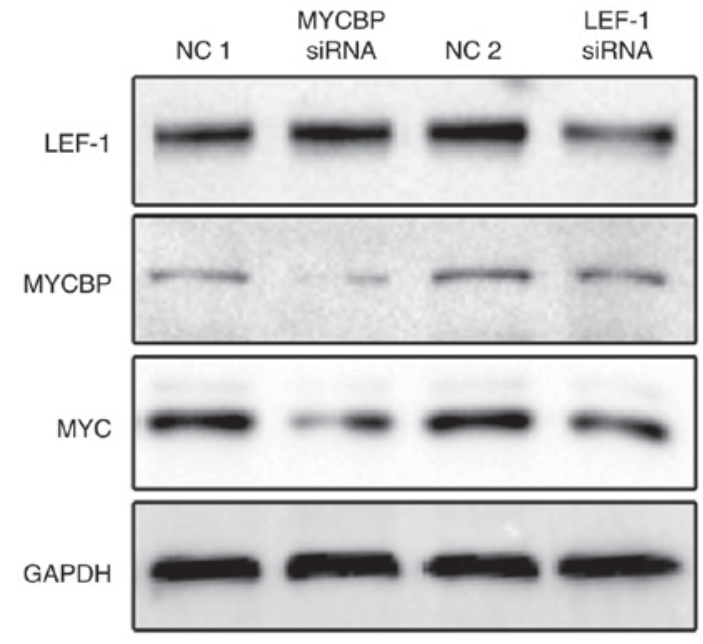

Figure 5. Transfection with MYCBP siRNA downregulated the protein expression of MYCBP and MYC in GC cells; transfection with LEF-1 siRNA induced a decrease in the protein levels of LEF-1, MYCBP and MYC in GC cells. GC, gastric cancer; MYCBP, MYC-binding protein; LEF-1, lymphoid enhancer-binding factor 1; NC, normal control; siRNA, small interfering RNA.

MYCBP may be a direct target of LEF-1. MYCBP was predicted to be a downstream target of LEF-1 (http://www. genecards.org/). To verify the results, western blotting was used to detect the expression of MYCBP following downregulation of LEF-1 expression. As shown in Fig. 5, in the MKN-45 cells, downregulation of LEF-1 with siRNA caused a marked reduction in MYCBP expression at the protein level. These findings indicated that MYCBP may be one of the direct targets of LEF-1.

\section{Discussion}

Recent studies indicated that MYCBP is abnormally expressed during tumorigenesis in numerous types of cancer. Jung and Kim (15) reported that the upregulation of MYCBP in colon carcinoma cells may have co-activating effects on MYC. Wang et al (20) reported that MYCBP regulated multiple biological processes within glioma cells, which is associated with malignant characteristics, including cancer cell proliferation, transformation and metastasis. Additionally, Furusawa et al (24) suggested that MYCBP is a trigger in the tumorigenesis of chronic myeloid leukemia. However, the potential role and molecular mechanisms of MYCBP in GC remains unclear.

In the present study, MYCBP was significantly overexpressed in GC tissues compared with adjacent normal gastric mucosal tissues, which indicated that MYCBP might serve an oncogenic role in human GC. Additionally, the function of MYCBP in the GC cell line MKN-45 was investigated, and it was demonstrated that MYCBP downregulation suppressed the migratory and invasive capacities of the MKN-45 cells in vitro, thus indicating that MYCBP might serve a key role in the metastasis of GC.

MYCBP is an $11 \mathrm{kDa}$ protein that binds to the $\mathrm{N}$-terminal region of MYC and stimulates the activation of E-box-dependent transcription by MYC (25). Overexpression of MYC has been suggested to serve key roles in cell migration and invasion by promoting the expression of target genes (26). Previous studies have been performed to inhibit the expression of MYCBP, and thus the MYC pathway, and observe the resultant inhibition of cancer cell migration and invasion (20,27). Furthermore, the overexpression of MYCBP facilitated Hedgehog signaling responses, whereas the knockdown of MYCBP compromised Hedgehog signaling responses (28). It has been suggested that abnormal Hedgehog signaling activity serves important roles in the development and progression of cancer, by promoting cell proliferation, mobility and invasiveness (29). Accordingly, blocking Hedgehog signaling has been demonstrated to inhibit the invasion and metastasis of cancer cells (30). As listed in GeneCards ${ }^{\circledR}$ : The Human Gene Database (http://www. genecards.org/), MYCBP is a member and positive regulator of the Notch signaling pathway. Previous studies concerning several cancer types demonstrated that blocking the Notch signaling pathway led to decreased expression and diminished bioactivity of urokinase-type plasminogen activator, which contributed to inhibited cancer cell migration, invasion and apoptosis $(31,32)$. A previous study confirmed that MYCBP acted downstream of the vascular endothelial growth factor receptor, and also indicated that when binding of MYCBP to cortactin was inhibited, tumor invasion, metastasis and angiogenesis were blocked (33).

Notably, the present results illustrated that transfection with MYCBP siRNA markedly induced $\mathrm{G}_{1}$ phase arrest in $\mathrm{GC}$ cells when compared with the control group. Recent research indicated that MYC also served a role in the cell cycle transition from $G_{1}$ to $S$ phase (34). The regulatory mechanisms underlying the cell cycle are complex, and any single pathway does not represent the full function of MYC. Hermeking et al (35) identified cyclin-dependent kinase 4 (CDK4) as a target of MYC, which functionally inactivates the products of the tumor suppressor genes RB and p16, providing a link between MYC and the CDK4/cyclin D1/retinoblastoma protein/p16 pathway, and may account for the lack of genetic alterations to retinoblastoma protein and p16 in certain types of cancer.

To investigate the possible function of MYCBP and to reveal its underlying molecular mechanism, the identification of regulatory targets is required. In MKN-45 cells in the present study, the downregulation of LEF-1 expression caused a reduction in the MYCBP protein level. This indicated that MYCBP may be one of the targets of LEF-1. LEF-1 has previously been reported to be expressed excessively and to function as an oncogene in numerous types of cancer (36). Furthermore, the TCF/LEF axis serves a crucial role in the Wnt/ $\beta$-catenin signaling pathway, and the Wnt signaling pathway has been shown to be involved in a number of cellular functions, including cell proliferation, survival, and differentiation $(37,38)$. Western blotting was used to detect the expression of MYCBP protein following the exogenous regulation of LEF-1 expression. On the basis of these results, MYCBP may be a target of LEF-1, which itself is an important factor of the Wnt signaling pathway.

In summary, the present study demonstrated that MYCBP is upregulated in GC tissues and cell lines, and is associated with the metastatic capacity of GC cells, potentially via interaction with LEF-1. These findings indicate that MYCBP may function as a novel tumor promoter in $\mathrm{GC}$, and may be a potential biomarker for the diagnosis of GC. To the best of the authors' knowledge, the present research is the first study to demonstrate that 
MYCBP is a downstream gene of LEF-1 of the Wnt signaling pathway in GC.

\section{Acknowledgements}

The present study was funded by the Medicine and Health Research Foundation of Zhejiang Province (grant no. 2017KY018) and the Zhejiang Provincial Natural Science Foundation of China (grant no. LY18H160043).

\section{Competing interests}

The authors declare that they have no competing interests.

\section{References}

1. Casaretto L, Sousa P and Mari J: Chemotherapy versus support cancer treatment in advanced gastric cancer: A meta-analysis. Braz J Med Biol Res 39: 431-440, 2006.

2. Torre LA, Bray F, Siegel RL, Ferlay J, Lortet-Tieulent J and Jemal A: Global cancer statistics, 2012. CA Cancer J Clin 65 : 87-108, 2015.

3. Zhang J, Zhan Z, Wu J, Zhang C, Yang Y, Tong S, Sun Z, Qin L, Yang $\mathrm{X}$ and Dong W: Association among polymorphisms in EGFR gene exons, lifestyle and risk of gastric cancer with gender differences in Chinese Han subjects. Plos One 8: e59254, 2013.

4. Xie Y, Zhi X, Su H, Wang K, Yan Z, He N, Zhang J, Chen D and Cui D: A novel electrochemical microfluidic chip combined with multiple biomarkers for early diagnosis of gastric cancer. Nanoscale Re Lett 10: 477, 2015.

5. Buiatti E, Palli D, Decarli A, Amadori D, Avellini C, Bianchi S, Bonaguri C, Cipriani F, Cocco P, Giacosa A, et al: A case-control study of gastric cancer and diet in Italy: II. Association with nutrients. Int J Cancer 48: 369-374, 2010.

6. Shi Y and Zhou Y: The role of surgery in the treatment of gastric cancer. J Surg Oncol 101: 687-692, 2010.

7. Dang CV: The interplay between MYC and HIF in the Warburg effect. Ernst Schering Found Symp Proc: 35-53, 2007.

8. Rabbitts PH, Watson JV, Lamond A, Forster A, Stinson MA, Evan G, Fischer W, Atherton E, Sheppard R and Rabbitts TH: Metabolism of c-myc gene products: c-myc mRNA and protein expression in the cell cycle. Embo J 4: 2009-2015, 1985.

9. Pelengaris $\mathrm{S}$ and Khan M: The many faces of c-MYC. Arch Biochem Biophys 416: 129-136, 2003.

10. Okuyama H, Endo H, Akashika T, Kato K and Inoue M: Downregulation of c-MYC protein levels contributes to cancer cell survival under dual deficiency of oxygen and glucose. Cancer Res 70: 10213-10223, 2010.

11. Mai S and Mushinski JF: c-MYC-induced genomic instability. J Environ Pathol Toxicol Oncol 22: 179-199, 2003.

12. Dang CV: MYC on the path to cancer. Cell 149: 22-35, 2012.

13. Hu D, Wu J, Tang X, Hu F, Yang Y, Du J, Ye S and Zhang R: Molecular cloning and tissue distribution of a Schistosoma japonicum gene encoding AMY-1. Mol Med Rep 4: 1267-1271, 2011.

14. Taira T, Maëda J, Onishi T, Kitaura H, Yoshida S, Kato H, Ikeda M, Tamai K, Iguchi-Ariga SM and Ariga H: AMY-1, a novel C-MYC binding protein that stimulates transcription activity of C-MYC. Genes Cells 3: 549-565, 1998.

15. Jung $\mathrm{HC}$ and Kim K: Identification of MYCBP as a beta-catenin/LEF-1 target using DNA microarray analysis. Life Sci 77: 1249-1262, 2005.

16. Chiurillo MA: Role of the Wnt/ $\beta$-catenin pathway in gastric cancer: An in-depth literature review. World J Exp Med 5: 84-102, 2015

17. Wu C, Zhuang Y, Jiang S, Liu S, Zhou J, Wu J, Teng Y, Xia B, Wang $R$ and Zou X: Interaction between Wnt/ $\beta$-catenin pathway and microRNAs regulates epithelial-mesenchymal transition in gastric cancer (Review). Int J Oncol 48: 2236-2246, 2016.

18. Sawa M, Masuda M and Yamada T: Targeting the Wnt signaling pathway in colorectal cancer. Expert Opin Ther Targets 20: 419-429, 2016.
19. Duchartre Y, Kim YM and Kahn M: The Wnt signaling pathway in cancer. Crit Rev Oncol Hematol 99: 141-149, 2015.

20. Wang H, Yan X, Ji LY, Ji XT, Wang P, Guo SW and Li SZ: miR-139 functions as an antioncomir to repress glioma progression through targeting IGF-1 R, AMY-1, and PGC-1 $\beta$. Technol Cancer Res Treat 16: 497-511, 2017.

21. Byun E, Park B, Lim JW and Kim H: Activation of NF- $\kappa$ B and AP-1 mediates hyperproliferation by inducing $\beta$-catenin and c-Myc in Helicobacter pylori-infected gastric epithelial cells. Yonsei Med J 57: 647-651, 2016.

22. Washington K: 7th Edition of the AJCC cancer staging manual: Stomach. Ann Surg Oncol 17: 3077-3079, 2010.

23. Livak KJ and Schmittgen TD: Analysis of relative gene expression data using real-time quantitative PCR and the 2(-Delta Delta C(T)) method. Method 25: 402-408, 2001.

24. Furusawa M, Onishi T, Taira T, Iguchi-Ariga SM and Ariga H: AMY-1 is a trigger for the erythrocyte differentiation of K562 cells. Int J Oncol 16: 339-345, 2000.

25. Sakamuro D and Prendergast GC: New Myc-interacting proteins: A second Myc network emerges. Oncogene 18: 2942-2954, 1999.

26. Dhru HD, McDonough Winslow WS, Armstrong B, Tuncali S, Eschbacher J, Kislin K, Loftus JC, Tran NL and Berens ME: Reciprocal activation of transcription factors underlies the dichotomy between proliferation and invasion of glioma cells. Plos One 8: e72134, 2013.

27. Jiang X, Hu C, Arnovitz S, Bugno J, Yu M, Zuo Z, Chen P, Huang H, Ulrich B, Gurbuxani S, et al: miR-22 has a potent anti-tumour role with therapeutic potential in acute myeloid leukaemia. Nat Commun 7: 11452, 2016

28. Lin C, Yao E, Wang K, Nozawa Y, Shimizu H, Johnson JR, Chen JN, Krogan NJ and Chuang PT: Regulation of Sufu activity by $\mathrm{p} 66 \beta$ and Mycbp provides new insight into vertebrate Hedgehog signaling. Genes Dev 28: 2547-2563, 2014.

29. Liao X, Siu MK, Au CW, Wong ES, Chan HY, Ip PP, Ngan HY and Cheung AN: Aberrant activation of hedgehog signaling pathway in ovarian cancers: Effect on prognosis, cell invasion and differentiation. Carcinogenesis 30: 131-140, 2009.

30. Feldmann G, Dhara S, Fendrich V, Bedja D, Beaty R, Mullendore M, Karikari C, Alvarez H, Iacobuzio-Donahue C, Jimeno A, et al: Blockade of hedgehog signaling inhibits pancreatic cancer invasion and metastases: A new paradigm for combination therapy in solid cancers. Cancer Res 67: 2187-2196, 2007.

31. Shimizu M, Cohen B, Goldvasser P, Berman H, Virtanen C and Reedijk M: Plasminogen activator uPA is a direct transcriptional target of the JAG1-Notch receptor signaling pathway in breast cancer. Cancer Res 71: 277-286, 2011.

32. Raghu H, Gondi CS, Dinh DH, Gujrati M and Rao JS: Specific knockdown of uPA/uPAR attenuates invasion in glioblastoma cells and xenografts by inhibition of cleavage and trafficking of Notch-1 receptor. Mol Cancer 10: 130, 2011.

33. Hashimoto A, Hashimoto S, Ando R, Noda K, Ogawa E, Kotani H, Hirose M, Menju T, Morishige M, Manabe T, et al: GEP100-Arf6-AMAP1-cortactin pathway frequently used in cancer invasion is activated by VEGFR2 to promote angiogenesis. PLoS One 6: e23359, 2011.

34. Sakamuro D and Prendergast GC: New Myc-interacting proteins: A second Myc network emerges. Oncogene 18: 2942-2954, 1999.

35. Hermeking H, Rago C, Schuhmacher M, Li Q, Barrett JF, Obaya AJ, O'Connell BC, Mateyak MK, Tam W, Kohlhuber F, et al: Identification of CDK4 as a target of c-MYC. Proc Natl Acad Sci USA 97: 2229-2234, 2000.

36. Delaunay S, Rapino F, Tharun L, Zhou Z, Heukamp L, Termathe M, Shostak K, Klevernic I, Florin A, Desmecht H, et al: Elp3 links tRNA modification to IRES-dependent translation of LEF1 to sustain metastasis in breast cancer. J Exp Med 213: 2503-2523, 2016.

37. Schambony A and Wedlich D: Wnt-5A/Ror2 regulate expression of XPAPC through an alternative noncanonical signaling pathway. Dev Cell 12: 779-792, 2007.

38. Willert $\mathrm{K}$ and Jones KA: Wnt signaling: Is the party in the nucleus? Genes Dev 20: 1394-1404, 2006. 